\title{
A Survey Based ON EEG Classification
}

\author{
${ }^{1}$ M.Deepthi, ${ }^{2}$ dr.P.Kavipriya \\ ${ }^{1}$ M.E. (Embedded System), ${ }^{2}$ Assistant Professor \\ Department Of Ece \\ Sathyabama University, Chennai
}

\begin{abstract}
Electroencephalography (EEG) based brain Computer interface (BCI) needs efficient algorithms to extract discriminative features from raw EEG signals. The issue of selecting optimizing spatial spectral features is key to high performance motor imagery(MI) classification, which is one of the main topics in EEG-based brain computer interfaces. Some novel methods are used first which formulates the selection of features as maximizing mutual information between class labels and features. It then uses an efficient algorithms for pattern feature extraction frame work, to select an effective feature set. The results shows the classification accuracy obtained and is compared with the other existing algorithms
\end{abstract}

\section{KEY WORDS}

Brain Computer Interface, feature extraction, classification, optimization and accuracy

\section{INTRODUCTION}

Brain Computer Interface (BCI) system, also called a Brain Machine Interface(BMI) is a communication system for controlling the devices like computer by human intensions which depends on the signals representing intensional brain activities. The Recognition procedure involves the two processes called feature extraction and classification. Different types of mental tasks of the human have been investigated,design of BCI.Classification of the EEG signals associated with mental tasks plays an important role.Feature extraction is one of the important issue in the design of a BCI.

One of the technique very frequently for extracting discriminant spatial features is common spatial pattern (CSP) method.The CSP mainly works on the band pass filtered EEG.Simultaneously optimizing spatial spectral filters is needed for selecting effective features. The CSP algorithm computes the spatial filters that maximize the ratio of the variance of the data mentioned in one class to the variance of the data mentioned in the other class.Using efficient techniques to BCI,the high complexity of the human Brain and the signal-to-noise ratio in EEG signals prevents EEG-based BCI systems.

CSP maximizes one class and minimizes the other class covariance.The optimization problem of CSP is used to maximize one specific class covariance with the normalization of two class covariance.CSSP,by using concatenation it doubles the channels of EEG samples into the original ones.Solution of CSSP is given by generalizing eigenvalue decomposition,which is similar to CSP.CSSSP combines different time delayed signals using raw EEG signals.DFBCSP optimizes by solving two problems of generalized eigenvalue decomposition in a sequential way and alternative way.By using the CSP technique ISSPL optimizes the spatial filters.SWCSP optimizes DOI : $10.5121 /$ ijit.2016.5101 
spectral and spatial filters with positive coefficients sequential way and iterative way.FBCSP constructs filter banks and CSP algorithm is used for obtaining spatial filters from EEG signals.OSSFN derives the spatial filters with maximizing the mutual information,which is estimated by the probability density function.
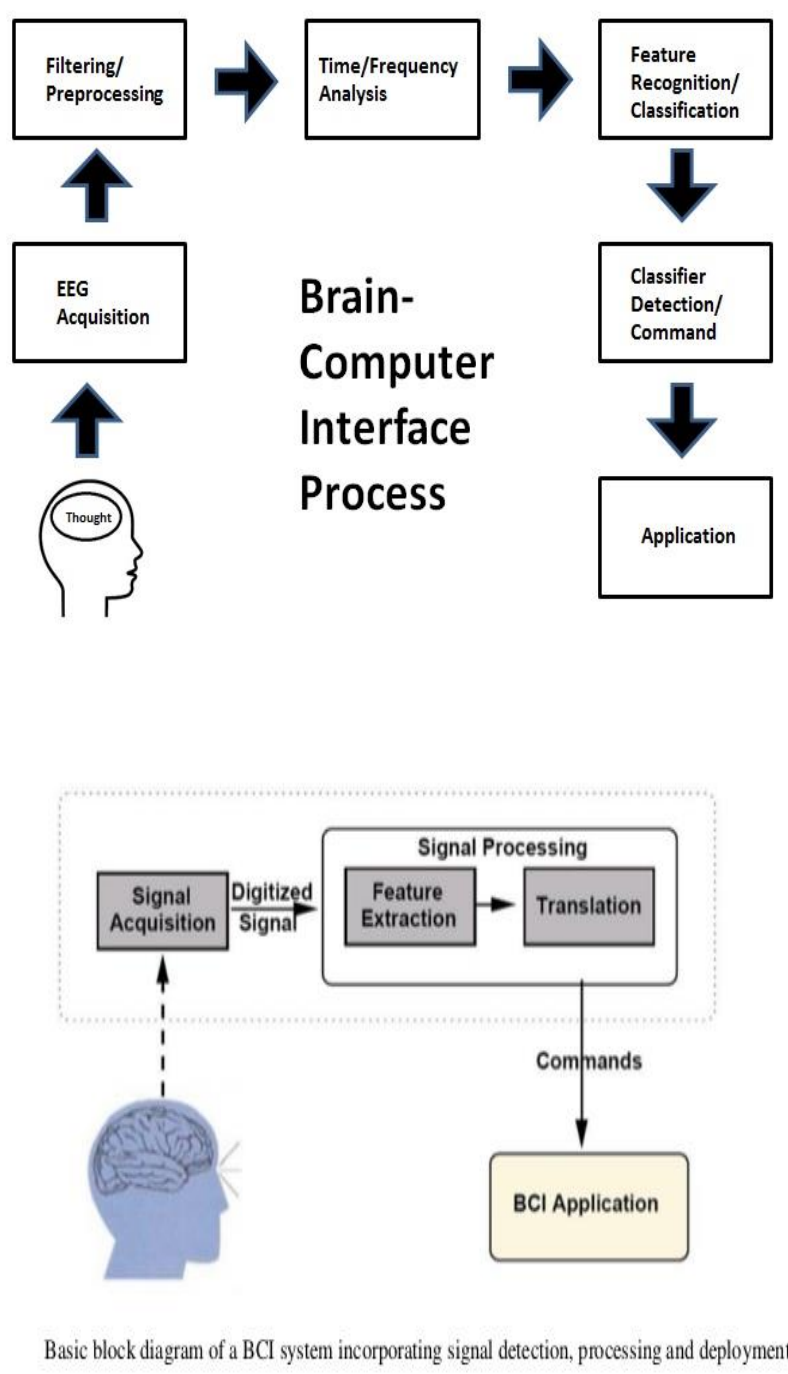

Fig(1): Block Diagrams

\section{DifFERENT METHOdOLOGY}

There are different methods used for the better classification of EEG signals. 


\subsection{Learning Algorithm For The Spatio Spectral Filtering}

Haihong Zhang, Zheng Yang Chin, Kai Keng Ang(2011), This paper deals with the FBCSP spatial filters.

Input: Training EEG data that consists of $\mathrm{N}$ sample blocks, each block will have a specific class label.

Output: The filtering network with optimized parameters for spatial filters, selection of the optimized band pass filter.

Step1: Construct an array of band pass filters that covers the EEG rhythms of motor imagery, then filter it.

Step2: For each band pass filter's output, construct a discriminative spatial filter space and compute co-variance matrices of two classes, compute eigen values and eigen vectors and sort them in a corresponding dimensions.

Step3: selection of optimum frequency band taken and set the optimized spectral filter and spatial filter.

\section{Mutual Information}
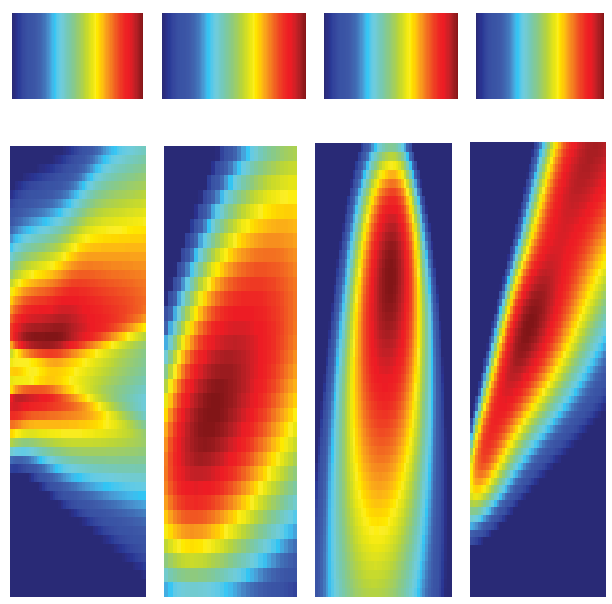

Fig(2):Subjects a,b,c,d

This figure shows the result for each four subjects in the BCI data set. Each pixel is representing a spatial filter, mutual information is denoted by color. According to their appearance some represents the bottom spatial filter from FBCSP and some corresponds to top filter from FBCSP. The surface is smooth that indicates gradient based optimization and the use of FBCSP spatial filters if for optimizing and initializing.

\subsection{Csp Algorithm}

Mahnaz Arvaneh, Cuntai Guan, Kai Keng Ang and Chai Quek(2011) deals with the CSP algorithm. 
The CSP aims at learning spatial filters, which maximizes the variance of band-pass filtered signals from different classes that corresponds to the power signals in the band and at achieving optimum discrimination on band power features.

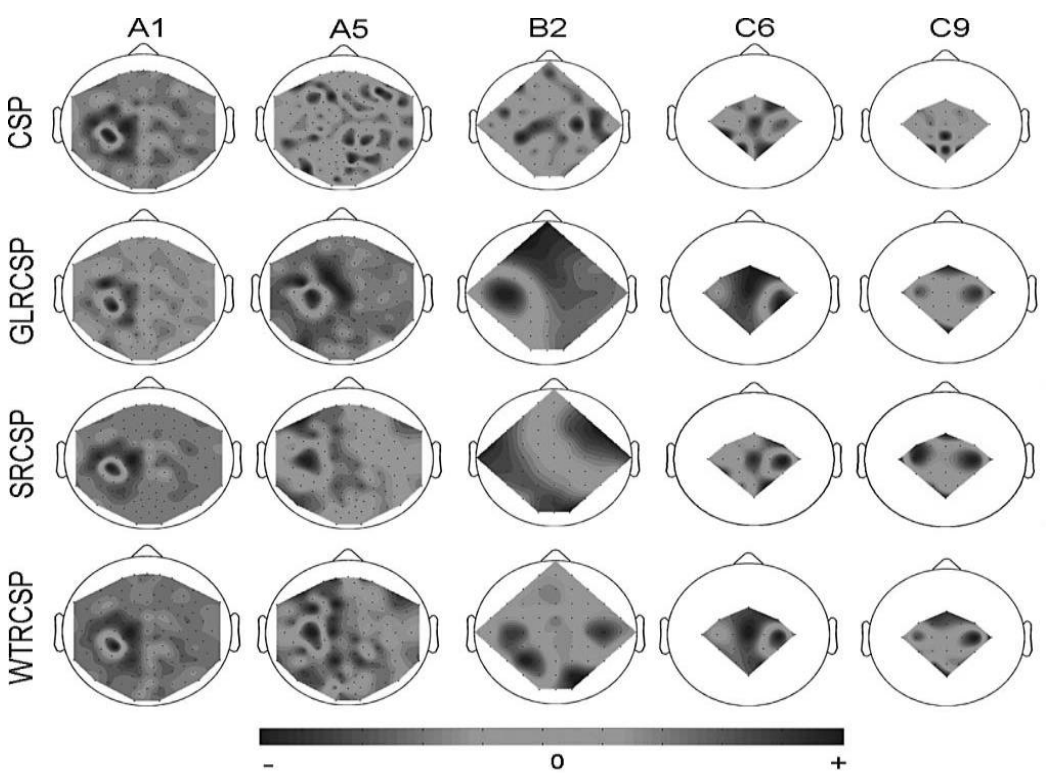

Fig.(3).Electrode weights for corresponding filters obtained with different CSP algorithms(CSP,GLRCSP,SRCSP)

Regularized CSP: RCSP belongs to CCSP family, which uses the data from the other subjects to get the co-variance matrix.The data from every subjects may not be used,it may be done only from the selected and relevant subjects.

Spatially RCSP:This algorithm is used to learn spatial filters and CSP ignores the spatial location of EEG electrodes.The SRCSP algorithm aims to make use of spatial information mainly to obtain spatially smoothed filters, in which filters electrodes will have similar weights.Electrodes has to measure similar brain signals, if they are close to each other.

CSP with Tikhonov Regularization(TR) or TRCSP:TR represents a classical format of regularization.It is used for the regression problems and consists in penalizing solutions with the large weights. The weighted Tikhonov RCSP is WTRCSP.

\subsection{Combination Of Wavelet Packet Transform, CSP, Mutual Information}

Behzad Amanpour and Abbas Erfanian (2013) deals with Wavelet Packet Transforms, CSP, Mutual Information.

Feature extraction is the main issue in the design of Brain-Computer I nterface.CSP is one of the 
popular discriminative feature technique in EEG.The CSP consists of the wavelet transform,which decomposes the EEG data of each channel.CSP is then applied on the reconstructed signals using each coefficient of wavelet.

Wavelet Packet: The wavelet transform is a popular and well-suited method for analyzing the irregular structures in signals. The wavelet transform can characterize the regularity of the signal by decomposing the signals which are localized in both the space and frequency. The CSP is applied on the reconstructed signal for each block due to the resolution properties of the wavelet transforms. Mutual Information Based Feature Selection: Optimal feature selection is possible properly and effectively through Mutual Information(MI).MI is used to measure the mutual dependence of two and more than two variables.If the mutual information is large between any two random variables it indicates that those two variables are related closely.If those two are independent,then the mutual information will be zero.

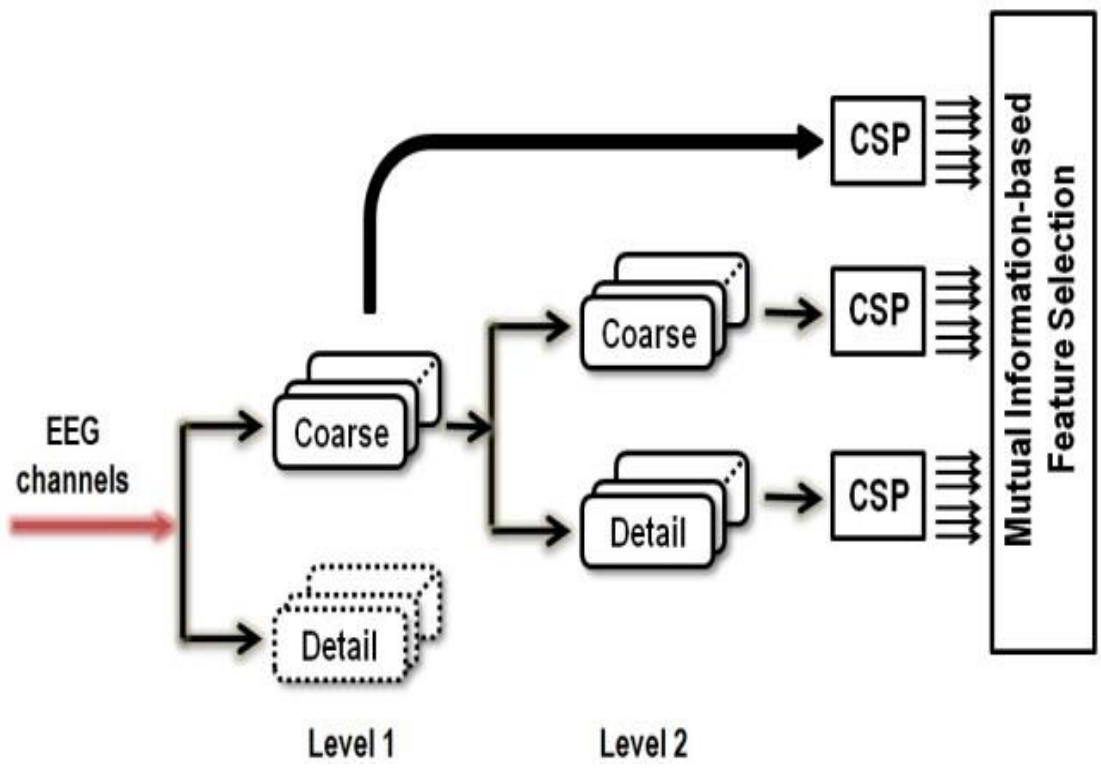

Fig(4):Diagram for Feature Extraction

CSP: The Common Spatial Pattern with Tikhonov Regularization is used to design the filters, which maximizes the variance of the filtering signal of one class and minimizes the other class. The popularity and efficiency of CSP is highly sensitive towards noise and is overfit in severe way to the small training data sets.

\subsection{LDA Algorithm}

Manojkumarmukul, Fumitoshi Matsuno(2010) deals with the Linear discriminant Classification.

The raw EEG signals has to be pre-processed by AMUSE algorithm,by doing this we will get three independent sources.AMUSE algorithm uses some principles to estimate components that should be de-correlated and less complex.The components are arranged in an decreasing order according 
to the covariance.AMUSE algorithm involves two step process.In $1^{\text {st }}$ step Principal Component Analysis(PCA) is applied on EEG brain signals, the $2^{\text {nd }}$ step is applied on lagged co-variance matrix of data obtained in the single step.Design of BCI system involves and contains five different categories of classifiers like linear classifier,neural networks,non-linear classifier,Bayesian classifier and nearest neighbour classifier and also combinations of these classifiers.Linear classifier is the popular algorithm for the design of BCI system. These classifiers are used to produce the boundary that seperates the two classes at a time.

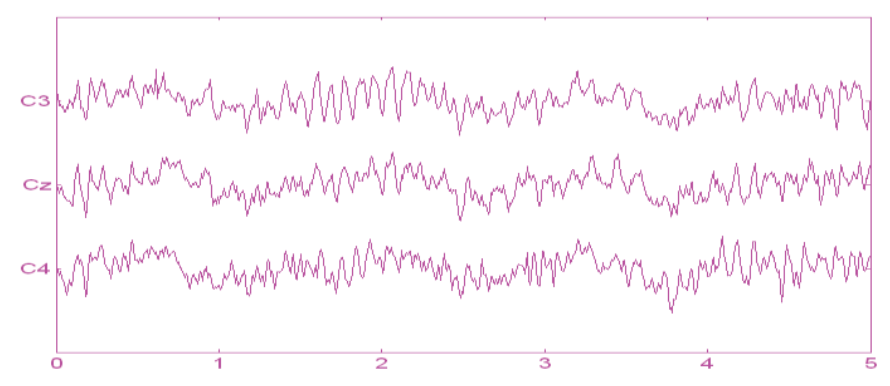

Fig(5):Recorded three channel EEG signals

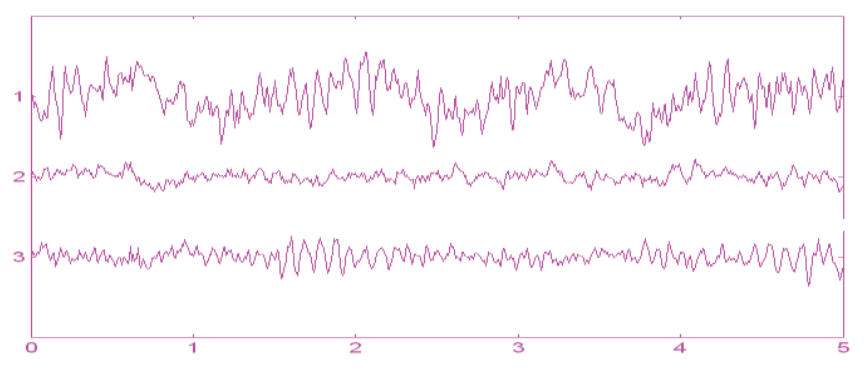

Fig(6):AMUSE filterd signal

Two pre-processing methods are used,one is of second order statistics based ICA AMUSE algorithm and the other is of linear FIR filters of order 16.Power spectrum estimation technique is used,the classification will be done by LDA.Design of the classifier is the main property to be done.After we know the frequency band information,LDA classifier has to be designed.BCI evaluation is denoted by the classification,accuracy,mutual information and information rate.The raw EEG signals are showed in the AMUSE filtered signal.

\section{CONCLuSion}

This paper(survey) proposed various methodologies which deals with the classification of EEG signals are analyzed.To reduce the dimensions of the raw EEG signals some optimization techniques are required.The classification accuracy,should be more using proper algorithms of data sets of BCI competitions. To improve the efficiency of the brain-computer interfaces better and suitable algorithms made to use. 
International Journal on Information Theory (IJIT) Vol.5, No.1, January 2016

\section{REFERENCES}

[1] J.Wolpaw,N.Birbaumer,D.McFarland, G.P.furtscheller,andT.Vaughan, "Brain-computer interfaces for communication and control", vol.113, no.6, pp.767-791, 2002.

[2] B. Blankertz, R. Tomioka, S. Lemm, M. Kawanabe, and K. R. Muller'Optimizing spatial filters for robust EEG single-trial analysis" IEEE Signal Process.Mag.,vol. 25, no.1, pp.41-56, 2008.

[3] H. Higashi and T. Tanaka,"Simultaneous design of fir filter banks and spatial patterns for the EEG signal classification”,IEEE Trans. Biomed. Eng.,vol. 60, no. 4, pp. 1100-1110, Apr. 2013.

[4] Haihong Zhang, Kai Keng Ang, Cuntai Guan,Chuanchu Wang, "Spatio-Spectral feature selection based on the robust mutual information estimate for brain computer interfaces",IEEE EMBS Minneapolis,2009.

[5] S.Park,E.Serpedin and K.Qaraqe,"Guassian assumption:The least favorable but the most useful,"IEEE Signal Process.Mag.,vol.30,no.3,pp. 183-186,may-2013.

[6] F.Lotte and C.Guan,"Regularizing common spatial patterns to improve BCI Designs:unified theory and new algorithms", IEEE Trans. Biomed. Eng., vol.58,no.2,pp.355-362,Feb-2011.

[7] R.Tomioka and K.R.Muller,"A Regularized Discriminative framework for EEG analysis with application to brain computer interface", Neuro-image, vol.49,no.1,pp.415-432,2010.

[8] H.Wang,"Multiclass filters by a weighted pairwise criteria for EEG single-trial classification",IEEE Trans. Biomed. Eng., vol.58, no.5, pp.1412-1420,Feb-2011.

[9] H.Suk and S.Lee,"A Novel Bayesian framework for discriminative feature extraction in brain-computer interfaces",IEEE pattern anal.mach.Intell.,vol.35,no.2,pp.286-299,Feb-2013.

[10] K.K.Ang, Z.Y.Chin, H.Zhang, C.Guan,"Mutual information-based selection of optimal spatial-temporal patterns for single-trial EEG-based BCIs,"pattern recog.,vol.45,no.6, pp.2137-2144,2012.

[11] J.Meng, G.Huang, D.Zhang and X.Zhu,'Optimizing spatial spectral patterns jointly with channel configuration for brain-computer interface,"Neuro computing,vol.104,pp.115-126,2013. 